\title{
Bipartite electronic SLA as a business framework to support cross-organization load management of real-time online applications
}

Stuart E. Middleton, Mike Surridge, Bassem I. Nasser, Xiaoyu Yang

\author{
IT Innovation Centre, University of Southampton, \\ 2 Venture Road, Southampton, SO16 7NP, UK \\ \{sem, ms, bmn, kxy\}@it-innovation.soton.ac.uk
}

\begin{abstract}
Online applications such as games and e-learning applications fall within the broader category of real-time online interactive applications (ROIA), a new class of 'killer' application for the Grid that is being investigated in the edutain@grid project. The two case studies in edutain@grid are an online game and an e-learning training application. We present a novel Grid-based business framework that makes use of bipartite service level agreements (SLAs) and dynamic invoice models to model complex business relationships in a massively scalable and flexible way. We support cross-organization load management at the business level, through zone migration. For evaluation we look at existing and extended value chains, the quality of service (QoS) metrics measured and the dynamic invoice models that support this work. We examine the causal links from customer quality of experience $(\mathrm{QoE})$ and service provider quality of business (QoBiz) through to measured quality of service. Finally we discuss a shared reward business ecosystem and suggest how extended service level agreements and invoice models can support this.
\end{abstract}

Keywords: SLA, business model, value chain, cross-organization, load management, ROIA, Grid

\section{Introduction}

As Grid technology matures [8] it raises the possibility of improving the way that online applications such as games and e-learning applications are provisioned and managed. The edutain@grid project [7] is investigating just this. This type of application needs resource provisioning that is secure, robust, scalable and flexible enough to support the value chains found in real-time online domains. As case studies within the edutain@grid project we have two distinct ROIAs, a real-time massively multiplayer online (MMO) game developed by Darkworks and an e-learning search and rescue training simulator developed by BMT Cordah. Through these case studies we aim to evaluate how Grid technology can support and provision ROIAs and their associated business relationships.

The online game market sector is growing, soon to be worth billions [5], and the elearning market is currently worth millions [10]. Analysis of the business relationships is key to developing a commercially viable supporting middleware. In edutain@grid we have implemented a business layer that flexibly supports complex 
value chains in a way where multi-organizational resource provision can scale massively and gracefully with ROIAs as they become more successful and attract more customers. Extending an existing business Grid middleware, GRIA [17], we make use of bipartite service level agreements (SLAs) and dynamic invoice models to encode business relationships. Although not the focus of this paper, our middleware supports single sign-on security, with X.509 credentials and Security Assertion Markup Language (SAML) access control tokens put in place prior to user's game play to avoid real-time performance costs.

This paper presents our novel business framework, using scalable dynamic bipartite service level agreements and invoice models based on quality of service. Our business level support for cross-hoster load management, though zone migration, is not currently seen with ROIA provisioning today. The concept of zone depends on the application and can be $3 \mathrm{D}$ areas in a game world, training scenarios etc. In addition to our proof of concept implementation we present a new shared reward business ecosystem that could help shape ROIA provisioning models as they grow in scale over the coming years.

\section{Related work}

Most Grid middleware systems such as the Globus toolkit [9], gLite [6], and UNICORE [3] have somewhat rigid infrastructures and are not very cost-effective at supporting changes to the basic business infrastructure associated with a dramatic scaling-up of service provision requirement. In edutain@grid our support for bipartite business relationships makes the provisioning network flexible and easy to grow over time. Supporting cross-hoster service provision and load management by design allows us to manage the changes in ROIA scale cost-effectively.

The use of service level agreements has been used as part of the paper management of supply chains and telecommunication services for decades. As service provision becomes more dynamic, with increasingly agile service composition, electronic service level agreement lifecycle management gains importance. A number of standardization attempts have been seen [13] but failed to gain traction within the community (e.g. WLSA, SLAng). Currently WS-Agreement [1] is the most widely adopted standard to represent service level agreements, but focuses on protocol and lacks detailed standards for representing quality of service metrics, constraints and penalties. Edutain@grid builds on this work defining bipartite service level agreements between coordinators and hosters to model our business relationship networks in a flexible way, and to set quality of service expectations from ROIA provisioning that can be measured and monitored.

The associated area of cloud computing has come about from an evolution of grids and service oriented architectures [18] and gained popularity when IBM and Google $[12,14]$ announced their collaboration. Clouds focus on virtualization coupled with time / CPU multiplexing, load balancing and multi-user service hosting to provide scalability. The cloud middleware hides the complexity involved in finding and preparing remote 'bare metal' computing resources. Cloud computing is a scalable solution but current implementations ignore geographic location (important for 
network performance), are single-hoster and lack support for dynamic service level agreements [4]. In edutain@grid we support load balancing between multiple hosters and use bipartite service level agreements to manage complex business relationships.

In the gaming space there are a number of existing commercial implementations of middleware for large scale 3D worlds supporting massive multiplayer online games. The Grid community has had some impact into this area with commercial offerings from Butterfly Grid [11] and BigWorld [2]. Butterfly Grid is based on the Globus toolkit and provides a peer to peer network of servers at a single hoster along with IP level security and single sign-on for in-game user accounts. BigWorld server provides single-hoster cluster management along with zone migration and bandwidth control via level of detail prioritization. Edutain@grid moves beyond these capabilities by supporting multiple hosters, and cross-hoster load management through zone migration, allowing massive scale-up to gracefully occur around successful ROIAs.

For the e-learning sector frameworks [16] have been developed using client-server, peer to peer and web service architectures but all suffer from associated poor scalability and fault tolerance / reliability. More recently Grid technology has been introduced in an attempt to bring in scalable distributed resources and allow elearning applications with higher resource demands to be developed cost-effectively. This Grid focus is on automated service composition and adaption. In edutain@grid we support e-learning applications with real-time performance criteria, a new aspect that has not been applied to e-learning Grids yet.

\section{Real-time online interactive application case studies}

The edutain@grid project includes two exemplar case study applications; an online multiplayer game and an e-learning multi-student training application. These applications have allowed the edutain@grid project to build a proof of concept architecture and test different aspects of our approach to ROIA provisioning. Figure 1 provides screenshots from these applications in action.

A massively scalable online 3D first person cooperative shoot-em-up game has been developed by Darkworks called 'Hunter'. This is a fast paced game with a massively scalable 3D hexagonal segmented play area that grows as new players connect. Key quality of service metrics are client packet latency $(<500 \mathrm{~ms})$ and server frame rate $(>15$ frames/sec). This pilot application is typical of massively multi-player online (MMO) first person perspective (FPS) games where games support 1000's of players from multiple geographic regions.

An e-learning shell application has been developed by BMT Cordah to run training applications such as their Search and Rescue (SAR) application within a multi-user voice over IP (VOIP) support environment. Supervisors and students remotely connect, and control is shared via a hot-seat protocol. The supervisor can monitor each student's progress as they participate in coast-guard role-play training simulations and communicate using video and audio. The application supports the Sharable Content Object Reference Model (SCORM) standard in common with most commercial e-learning applications. Sessions can involve up to 100 students and a 
few supervisors from multiple geographic regions. The key quality of service metric is data throughput to ensure acceptable VOIP performance during training sessions.
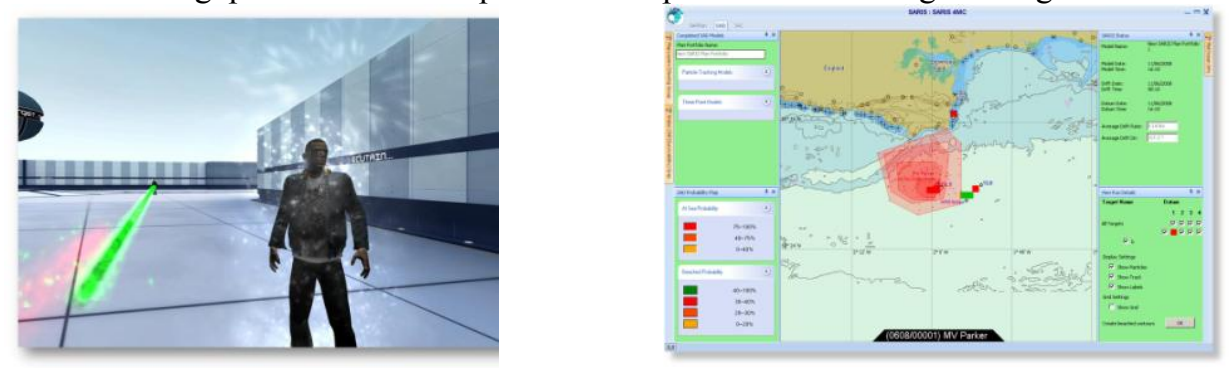

Fig. 1. Hunter online game and Search and Rescue (SAR) e-learning application screenshots

\section{Flexible business models suitable for ROIA}

The value chain for existing commercial ROIA provision is relatively simple, with a single service provider, or 'hoster', provisioning model underpinned by written fixed term service level agreements, between a customer and the hoster. This service level agreement defines the hardware that will be provided for the duration of the contract and cost to the customer. Penalties are often written in to compensate for failure of hardware availability or uptime. The scalability of this type of provisioning model is limited to the number of servers a hoster can provide. Vendor lock-in is a restriction for customers, and for ROIAs with large user-bases in many geographic regions multiple vendor agreements are often needed to ensure servers are geographically close to clients to increase communication performance.

In edutain@grid we have experimented with bringing Grid concepts to support more scalable multi-hoster value chains. We recognize that ROIA provision needs to start small, with an entry level low-cost single-hoster provision, and scale up gracefully through several orders of magnitude of users as a ROIA grows in success and popularity. We have introduced a third actor into the current commercial provisioning relationship, a broker or 'coordinator', that allows flexible value chains made up of many on-demand bipartite business relationships. The customer, or game player, is assigned a server provisioned by a hoster via the coordinator. We use ondemand electronic bipartite service level agreements to encode pricing and expected quality of service between the coordinator and hoster. User account management is provided by the coordinator for the customer. Hosters run a trade account service to record invoices for provisioned service and coordinators make use of existing customer payment models (e.g. PayPal).

Electronic on-demand service level agreements allow pricing based on measured quality of service and resource usage, not just hardware costs. This flexibility to pay for what is actually used allows coordinators to start small, sharing hoster resources with other coordinators. As users for a ROIA increase a greater share of each hoster's resource can be taken and new hosters brought in to provision the increased load. 
Edutain@grid supports cross-hoster zone migration allowing seamless load balancing between hosters with differing resource available from different geographic regions.

Supporting the electronic service level agreements in edutain@grid is a flexible invoice model that provides variable pricing, with cost components proportional to the quality of service measured, and banded pricing, where the overall cost is linked to bands based on quality of service threshold levels achieved by the provider. Classic invoicing components are also provided for cost per duration and penalty fees for breaches of quality of service thresholds. These invoice tools provide us with a flexible business layer that supports a variety of mechanisms to provide business incentives for key actors in the ROIA value chain.

\section{Case study : edutain@grid business layer architecture}

The edutain@grid business layer implementation supports the three phases of the service level agreement lifecycle, contract definition, negotiation and enforcement. For contract definition we have implemented a workflow, shown in figure 2. Multiple service level agreements can be setup for multiple coordinators and ROIAs providing a flexible and scalable bipartite value network.

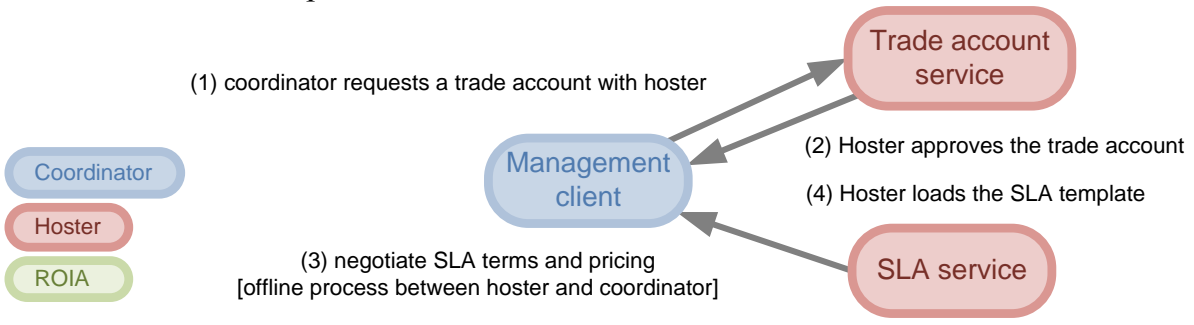

Fig. 2. edutain@grid SLA contract definition workflow

\begin{tabular}{|c|c|}
\hline \multicolumn{2}{|l|}{ SLA template [edutain@grid] } \\
\hline $\begin{array}{l}\text { Duration } \\
\text { Start time, End time, Currency [€] } \\
\text { Price per time unit [e.g. } 1 € \text { per day] }\end{array}$ & $\begin{array}{l}\text { Pricing term [accumulated QoS value] } \\
\text { Metric definition [URI, unit] } \\
\text { Price per unit of the accumulated value }\end{array}$ \\
\hline $\begin{array}{l}\text { Pricing term [peak QoS value] } \\
\text { Metric definition [URI, unit] } \\
\text { Price per unit of the peak value }\end{array}$ & $\begin{array}{l}\text { Pricing term [penalty value] } \\
\text { Metric definition [URI, unit] } \\
\text { Threshold values [upper, lower] } \\
\text { Penalty price if threshold breached }\end{array}$ \\
\hline $\begin{array}{l}\text { example values from edutain@grid SLA } \\
3 \text { month duration, } 1 € \text { per day, billed every month }\end{array}$ & $\begin{array}{l}1 € \text { cost per accumulated client connection count } \\
\text { penalty } 20 € \text { if upper client packet latency }>100 \mathrm{~ms}\end{array}$ \\
\hline
\end{tabular}

Fig. 3. edutain@grid XML SLA template outline

The edutain@grid service level agreement XML template structure, figure 3, contains sections for static hardware provision, cost for duration of provision, variable cost components based on quality of service measurement and penalties based on breaches of agreed thresholds. We use metrics for server packet latency (ms), packet loss (\%), data throughput (bytes/s), server tick time (ms) and client connection count. 
We have implemented a discrete offer protocol for contract negotiation (figure 4) in addition to session management; hosters have local provisioning sessions and coordinators have global sessions to manage collections of local session. More complex multi-stage negotiation strategies are possible but not cost effective for the value of individual provisioning contracts (typically €100's for a few months).

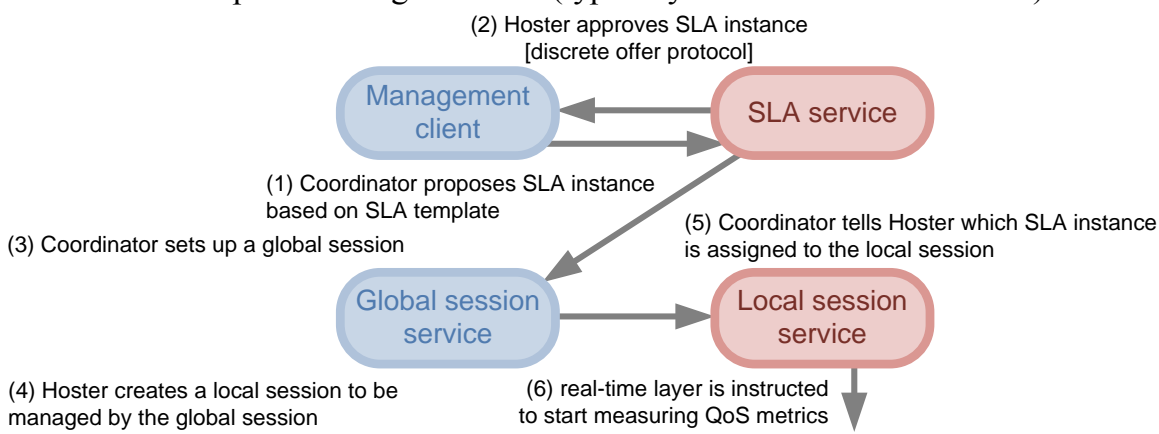

Fig. 4. edutain@grid SLA contract negotiation workflow

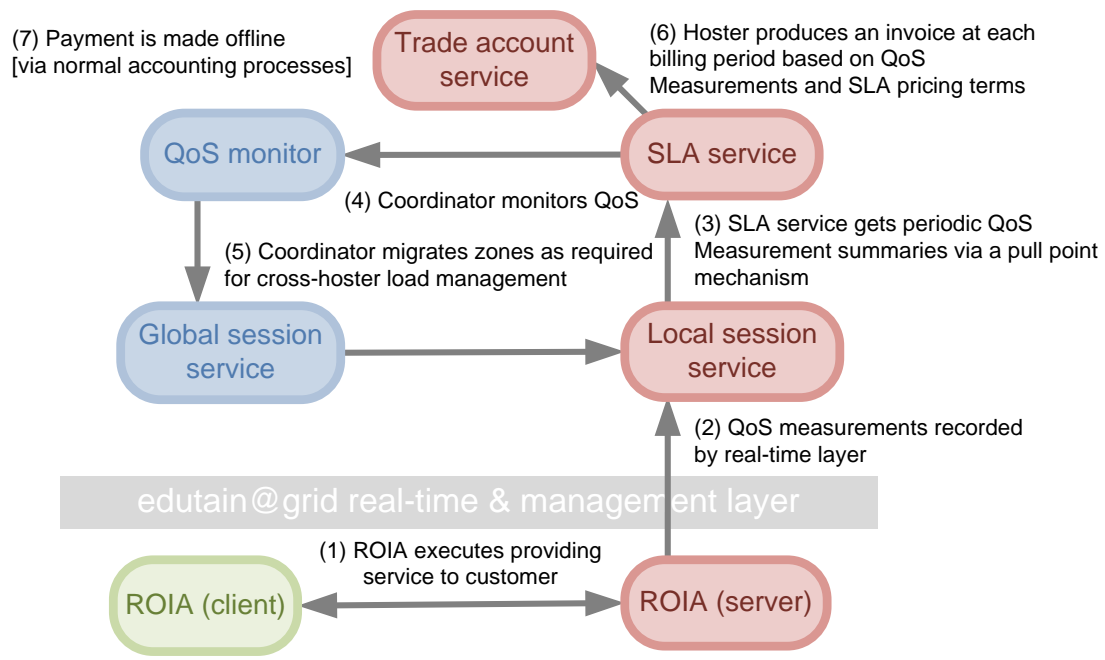

Fig. 5. edutain@grid contract enforcement workflow

The final step is contract enforcement, shown in figure 5, where users join ROIA sessions and quality of service metrics are recorded for the duration of each session runtime. The coordinator will monitor quality of service levels and can choose to (manually and/or automatically) migrate zones from one hoster's session to another, allowing cross-hoster load balancing. Edutain@grid thus implements business level control over real-time hoster to hoster zone management, something not seen in ROIAs today. The invoice is based on the terms in the service level agreement and actual payment by the coordinator to the hoster is made via normal accounting procedures. 


\section{Evaluation beyond Quality of Service for ROIA provision}

If we look at the whole business eco-system [15] we see that quality of service (QoS) are objective facts that are measureable, but what really matters to actors in the ecosystem depends on their perspective. The customer is primarily interested in the quality of experience (QoE) that good quality of service allows, ensuring the ROIA delivers as expected. The coordinator and hoster are interested in the quality of business (QoBiz), in particular the value gained for doing their role in service provision. If the QoE and QoBiz are causally linked to measureable QoS then the business ecosystem as a whole should be able to prosper.

From the customers perspective QoE for a game is linked to the ability to connect to a server, play with friends, ease of connection and use of the ROIA and the lack of any game perceivable game lag. For an e-learning application QoE means the ability to connect to server, talk to the supervisor and the quality of coaching received. There is a relatively clear cause and effect from QoE to the key QoS metrics. The data throughput will affect the ability of students to understand the supervisor via VOIP. The server frame rate and client packet latencies will affect game lag. Simple single sign-on security ensures easy login.

The impact QoE has on the value chain is on customer repeat business and the likelihood of attracting new business through word of mouth. These effects will impact future customer numbers, and thus the value of the overall business proposition for a ROIA provision network.

From the coordinator and hosters perspective the QoBiz comes down to the revenue obtained from the business proposition. Each decision they must make is done so in the context of how it will affect their QoBiz. For hosters key decisions are:

- will they accept new load

- will they signal to the coordinator they are (or might be) having trouble provisioning existing load

- $\quad$ are they able to shift internal resource to ensure QoS for existing load

- how much to charge a coordinator for provision of service.

For coordinators key decisions are :

- which hoster (who, where) should receive new load

- when, where and who to migrate ROIA load cross-hoster

- if, when and where a new hoster should be brought into the scalable value network for a specific ROIA

- how much to charge a customer for using a ROIA

- how much to pay hosters for service provision.

In order to ensure good QoBiz pricing incentives must be associated with each key business decisions and ultimately causally linked back to the final customers QoE. In this way a value chain and associated business model is setup so that all stakeholders are incentivised to increase overall QoBiz. Figure 6 shows the business ecosystem from a QoBiz perspective, showing actors and how revenue flows between them.

We have investigated within the edutain@grid business layer implementation pricing instruments for hardware prices for a duration, variable prices per quality of service measurement, penalty costs for quality of service breaches and price banding.

A hardware cost per duration incentivises the hoster to accept load at every opportunity. A penalty cost reduces the incentive to under-provision and provides a 
basic incentive framework in which ROIAs can be provisioned. However there is no incentive for the hoster to work to provide better quality of experience, or help grow the quality of business; the only incentive is to provide momentary quality of service on a case by case basis.

Introducing variable pricing based on measured quality of service allows us to define customer focused metrics such as the number of client connections, server frame rate, client connection latency and data throughput. Banded pricing provides an increasing scale of penalty for bad quality of service and helps to discourage systematic under-provisioning that would otherwise be in the hosters interest since it would ensure resources are fully loaded at all times. These incentives link hoster provisioning to factors that affect customer quality of experience. It is up to the coordinator to select carefully the key quality of service metrics that really do have a causal link back to QoE; this might be difficult if the causal link is not clear. Improving QoE is likely to indirectly improve the QoBiz, via long term return business, so the coordinator is well motivated to ensure this.

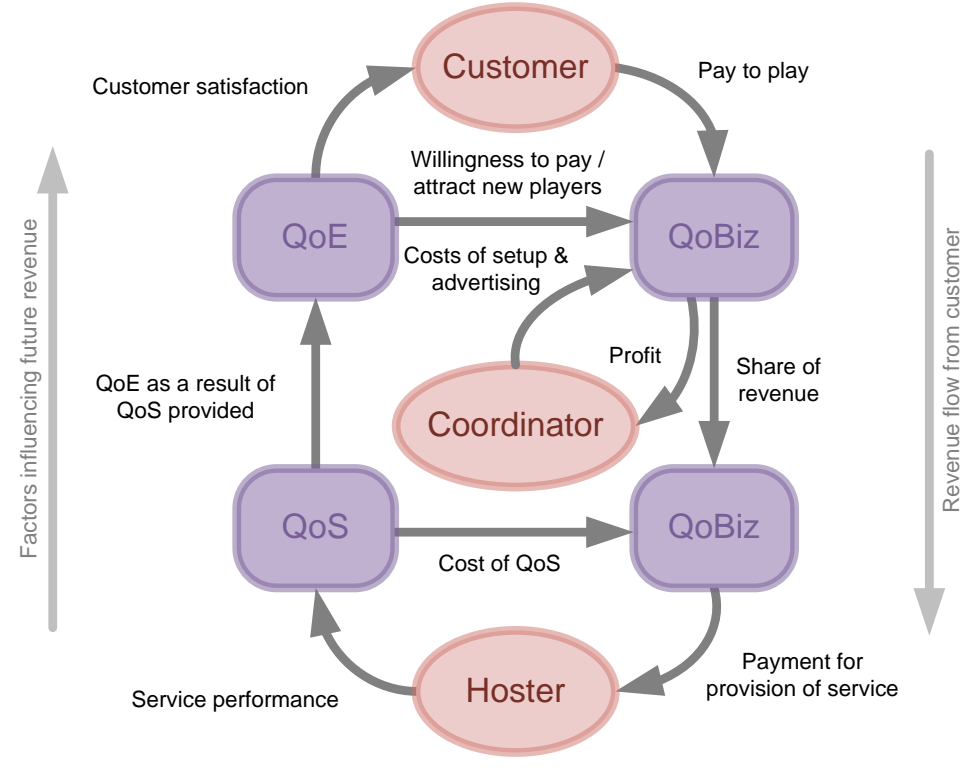

Fig. 6. Business ecosystem for actors in the ROIA value chain

We have found from experience in edutain@grid that these techniques are the limit with which the service and ROIA providers are really commercially comfortable, being not too far from the existing single-hoster fixed contract provisioning models that work commercially today. These pricing instruments do not, however, give hosters any direct incentive to work together to that ensure cross-hoster QoE is maintained.

We envisage a further enhancement to this incentive framework where the revenue from customers is directly shared, via coordinators, with the hosters. This shared value network has the advantage that hosters have a direct incentive to see the QoBiz grow. A service level agreement could define variable quality of service rewards in terms of a percentage of the revenue obtained from each customer, with a banded 
reward adjustment based on a longer term business metrics such as player number growth or increased coordinator income. Such shared rewards should encourage a limited degree of cooperation between hosters, encouraging proactive load sharing for under-provisioned hosters. Figure 7 shows what a shared incentive service level agreement might look like.

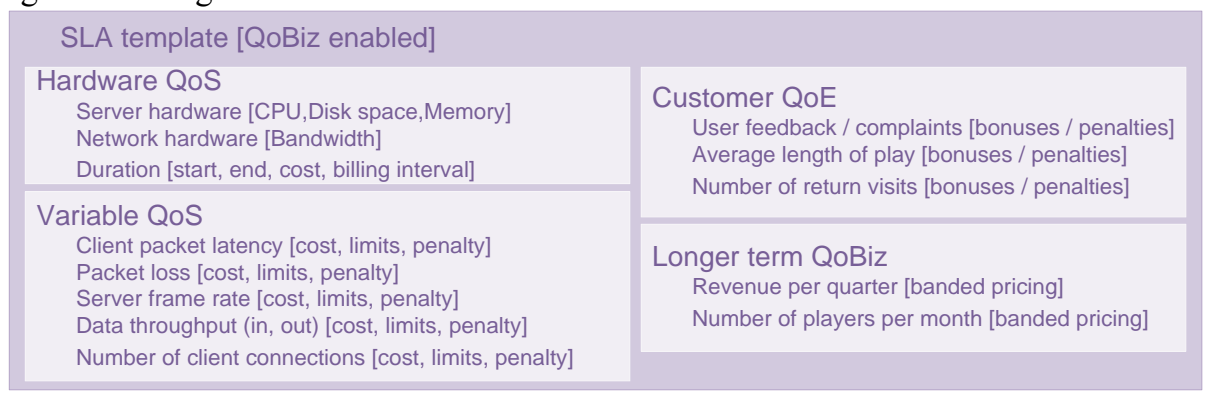

Fig. 7. Example QoBiz enabled SLA template

\section{Conclusions}

The edutain@grid business layer implements a scalable bipartite value chain, underpinned by electronic service level agreements, which can scale gracefully as small ROIAs with low numbers of users grow by several orders of magnitude to large successful ROIAs. We support invoice models that provides variable pricing, banded pricing, cost per duration and penalty fees. These invoice tools provide us with a flexible business layer that supports a variety of incentive mechanisms for key actors in the ROIA value chain.

The edutain@grid project includes two exemplar case study applications; an online multiplayer game and an e-learning multi-student training application. Key quality of service metrics are client packet latency, server frame rate and data throughput. We implement these applications as proof of concept demonstrators. Our business layer implementation supports contract definition using XML service level templates to define pricing and metrics. Contract negotiation follows a discrete offer protocol and contract enforcement is provided by continuous quality of service monitoring, ondemand cross-hoster zone migration and flexible invoice models.

We evaluate our value chains and incentive models in the context of both quality of experience and quality of business. We suggest setting up enhanced incentive models that share rewards between coordinators and hosters, providing a reason for hosters to cooperate with coordinators on cross-hoster load balancing. We show that whilst this is technically achievable the real question to be answered is will an evolving ROIA market see sufficient commercial benefits to make adoption worthwhile.

Acknowledgments

The work described in this paper is supported by the European Union through EC IST Project 034601 'edutain@grid'. 


\section{References}

1. Andrieux, A. et al,: Web Services Agreement Specification (WS-Agreement). 17 March 2007. http://www.ogf.org/documents/GFD.107.pdf (2007)

2. Big World Technology. http://www.bigworldtech.com/ (2002)

3. Breuer, D., Erwin, D., Mallmann, D., Menday, R., Romberg, M., Sander, V., Schuller, B., Wieder, P.: Scientific Computing with UNICORE. In Procs of NIC Symposium 2004, Dietrich Wolf, Gernot Münster, Manfred Kremer (Eds), John von Neumann Institute for Computing, Jülich, NIC Series, vol 20, pp 429-440 (2003)

4. Buyya, R., Yeo, C.S., Venugopal, S. : Market-oriented cloud computing: Vision, hype, and reality for delivering it services as computing utilities. Proceedings of the 10th IEEE HPCC08, IEEE CS Press, Los Alamitos, CA, USA (2008)

5. Cai, Y.: Networked Gaming: Driving the Future II. Industry report from Parks Associates (2009)

6. Czajkowski, K., Ferguson, D. F., Foster, I., Frey, J., Graham, S., Sedukhin, I., Snelling, D., Tuecke, S., Vambenepe, W.: The WS-Resource Framework (2004)

7. Fahringer, T., Anthes, C., Arragon, A., Lipaj, A., Müller-Iden, J., Rawlings, C., Prodan, R., Surridge, M.: The Edutain@Grid Project. GECON 2007, Rennes, France, August 28, 2007, pp 182-187. Lecture Notes in Computer Science, Springer Berlin / Heidelberg (2007)

8. Foster, I., Kesselman, C. (eds) : The Grid2: Blueprint for a New Computing Infrastructure. Morgan Kaufmann Publishers Inc., Elsevier, Boston, 2nd edition (2004)

9. Foster, I., Kesselman, C.: Globus: A Metacomputing Infrastructure Toolkit. International Journal Supercomputer Applications, 11 (2), pp115-128 (1997)

10.Helmer, J.: The UK e-Learning Market. Market report commissioned for Learning Light Limited (2007)

11. IBM : Butterfly.net: Powering Next-Generation Gaming with On-Demand Computing (2002)

12.IBM: Google and IBM Announced University Initiative to Address Internet-Scale Computing Challenges, Oct 8 (2007)

13.Kotsokalis, C.: What's in a Service Level Agreement?. SOA@SOI article (2009)

14.Lohr, S.: Google and I.B.M. Join in 'Cloud Computing' Research. NY Times, Oct 8 (2007)

15.van Moorsel, A.: Metrics for the Internet Age : Quality of Experience and Quality of Business. In Fifth Performability Workshop, Germany (2001)

16.Pankratius, V., Vossen, G.: Towards E-Learning Grids: Using Grid Computing in Electronic Learning. In Proc IEEE Workshop on Knowledge Grid and Grid Intelligence, Canada (2003)

17.Surridge, M., Taylor, S., De Roure, D., Zaluska, E.: Experiences with GRIA — Industrial Applications on a Web Services Grid. In Proceedings of the First International Conference on e-Science and Grid Computing, pp. 98-105. IEEE Press (2005)

18.Vouk, M.A. : Cloud computing-Issues, research and implementations. International Conference on Information Technology Interfaces, ITI 2008 (2008) 